\title{
Designing Fall Sequences That Minimize Robot Damage in Robot Soccer*
}

\author{
Javier Ruiz-del-Solar ${ }^{1}$, Rodrigo Palma-Amestoy ${ }^{1}$, Paul Vallejos ${ }^{1}$, R. Marchant ${ }^{1}$, \\ and P. Zegers ${ }^{2}$ \\ ${ }^{1}$ Department of Electrical Engineering, Universidad de Chile \\ ${ }^{2}$ College of Engineering, Universidad de Los Andes, Chile \\ jruizd@ing.uchile.cl
}

\begin{abstract}
In this paper is proposed a methodology for the analysis and design of fall sequences that minimize robot damage. This methodology minimizes joint/articulation injuries, as well as damage of valuable body parts (cameras and processing units). The methodology is validated using humanoid Nao robots and a realistic simulator. The obtained results show that fall sequences designed using the proposed methodology produce less damage than standard, uncontrolled falls.
\end{abstract}

\section{Introduction}

In soccer, as in many other sports that allow contact among players, it is usual that players fall down, as consequence of fouls, collisions with other players or objects, or extreme body actions, such as fast movements or ball kickings from unstable body positions. In addition, soccer players can intentionally fall down to block the ball trajectory (defense player) or to intercept the ball (goalkeeper). Therefore, we can affirm that the management of falls - e.g. how to avoid an unintentional fall, how to fall without damaging the body, how to achieve fast recovering of the standing position after a fall - is an essential ability of good soccer players. In general, this is also true for any physical human activity.

Given the fact that one of the RoboCup main goals is allowing robots play soccer as humans do, the correct management of falls in legged robots, especially in biped humanoid robots, which are highly unstable systems, is a very relevant matter. However, to the best of our knowledge this issue has almost not been addressed in the RoboCup and other mobile robotics communities. The current situation in the RoboCup is that: (i) In case of an unintentional fall, the standard situation is that robots do not realize they are falling down. Therefore, they do not perform any action for diminishing the fall damage. After the fall, they recognize they are on the ground using their internal sensors, and they start the standing up sequence of movements. There are some few examples of systems in the literature that detect unstable situations and avoid the fall [1][4][5][6]; (ii) In most of the cases, unintentional or intentional falls, robots fall as deadweight, without using a fall sequence that can

* This research was partially supported by FONDECYT (Chile) under Project Number 1061158. 
allow them to dissipate some of the kinetic energy of the fall or to protect some valuable body parts, as humans do. Two of the few works that address this issue are [2][3], although none of them was developed in the context of RoboCup; and (iii) The damage of robot components or environments parts after a fall is a real problem. This is one of the reasons for limiting the size of robots in some RoboCup leagues (e.g. RoboCup TeenSize league).

In this context, the aim of this paper is to address the management of falls in robot soccer. In concrete, we propose a methodology for designing fall sequences that minimize joint/articulation injuries, as well as the damage of valuable body parts (cameras and processing units). This fall sequences can be activated in case of an intentional fall or in case of a detected unintentional fall. It is not our intention to cover exhaustively this topic in this article, but to focus the attention of the community into this important problem. This paper is organized as follows. In section 2, some related work about human's fall studies is presented. The analysis of these studies suggests some guidelines for the management of falls in robots. The here-proposed methodology for designing fall sequences that minimize the robot damage is described in section 3 . In section 4 some experimental results of the application of this procedure in Nao robots are presented. Finally, in section 5 some conclusions of this work are given.

\section{Related Work}

Uncontrolled falls in biped robots have been largely ignored except for some very interesting works such as [2][3][7]. In general, falls are characterized by violent impacts that quickly dissipate and transfer important amounts of kinetic energy through joints, bones, and tissues. Falling looks like a dual event of walking. Avoiding falls gives a complementary view to walking, which is a controlled fall. In this section, we will analyses the literature about human's falls, which can give us some insights on how to manage falls in robots. There are several approaches to study falls in humans: what has been reported in medical literature, the studies done by people in biomechanics, the techniques developed in martial arts, and the efforts of the animation industry. These will be here reviewed.

Medical Studies. In [8] a fall is defined as "an unintentional event that results in a person's coming to rest on the ground or on another lower level." Falls are in general the result of the convergence of several intrinsic (muscle weakness, visual deficit, poor balance, gait defects, etc.), pharmacological (walking under the influence of alcohol, being under strong medication, etc.), environmental (uneven terrain, poor lighting conditions), and behavioral related (daily tasks, sports, violence, etc.) factors $[10][11][12]$. The rapid transmission of forces through the body that follow an impact against a surface cause injuries according to the energy-absorbing characteristics of the surfaces that receive the impact, the magnitude and direction of the forces, and the capacity of the tissues to damage [11]. Fall prevention in human has focused on balance and gait impairments, which are mainly affected by the interaction of the sensory (ability to determine whether the center of gravity of the body is within the support of the body or not), neuromuscular (transmission speed of the nervous impulses), and musculoskeletal systems (the available muscular force determine the range of possible movements), and their integration by the central nervous system (Parkinson's disease). 
In general, falls from a standing height produce forces that are one order of magnitude greater than those necessary to break any bone of an elderly woman [11]. However, approximately no more than $10 \%$ of falls in older people cause fractures [10][11]. The most common examples of serious injuries, besides tissue and organ damage, are hip and wrist fractures [11]. This is a clear indicator that people constantly use fall-handling strategies that manage to reduce injury-producing falls. Wrist injuries are also interesting because they indicate an active intent of people to stop their falls using limbs to shift the impact away to less important organs or bones.

Biomechanical Approach. Even though medical literature has been studying falls for several decades, the needs posed by high efficiency sports and the possibilities created by technological advances such as motion capture equipments has spawned new approaches to the science of human movement, also called biomechanics. This has made possible to understand human dynamics with greater detail and to generate more precise mathematical models of this biological machine: (i) The musculoskeletal system is now modeled as a combination of something that exerts the force, a spring, and a damping system [13][14]; (ii) Machine learning approaches have been used to classify movements in order to understand their relationships, and to prove the existence of clusters of movement patterns [15]; (iii) It has been possible to determine the role of the center of mass of the body in all types of movements, i.e. rock climbing [16]; (iv) Another important aspect are interactions with external objects, where, for example, it has been possible to determine that people require several minutes to adjust their movements to changing asymmetrical loads [17][18], or how vision and limbs coordinate to follow and manipulate balls while juggling [19]; and (v) Control of synchronization between many people exhibiting rhythmic movements under some conditions has been proven to be independent from force control [20].

Of special interest, from the point of view of studying falls, are studies of people displaying fast interactions with the ground or objects. Of special interest is the study of Gittoes et al [21], which proves that soft tissue strongly contributed to reduce loading when landing on the ground. Other studies of voluntary fast transitions show that it is possible to generate complex pattern activation patterns that allow to control sudden movements with an amazing degree of control [22][23][24][25].

Martial Arts. In the previous paragraphs we have pointed out the biological aspects of falling. But, Is it possible to control a fall in order to minimize damage? Is it possible to modify a fall in order to achieve some dynamic objective? These questions have been answered long ago by martial arts (check [26]). Of these, Judo and Taekwondo, are perfect examples. Both disciplines teach how to fall from different positions: forward, backward, and sideways. All these techniques are extremely effective in the sense that produce a sequence of movements that vary the geometry of the human body in order to lower the force of the impacts, and spread the kinetic energy transfer through a wider contact area, a longer lapse of time, and limb movement. Moreover, some of these techniques are designed to allow the fighter to move away from the attacker and prepare himself to continue the combat by quickly recovering an upright stance.

Human Dynamics Simulation. The constant pressure of the computer animation market for more realistic special effects has spurred a lot of research in this topic in the last decades [27][28][29]. This research has even tackled problems not studied by other disciplines, such as the reproduction of destructive movements that are 
impossible to study in humans due to their nature [30][31]. Given that the tools created by this industry are completely located in simulated environments, many of them naturally produce very realistic falls with physically plausible kinematics and dynamical interactions.

Discussion. In general, human falls are characterized by unexpected impacts that affect the whole body. Overall, a fall should not be analyzed as a very local impact. On the contrary, its forces propagate along the entire body and help to distribute and dissipate its effects. In general falls can be classified into three cases according to the degree of awareness of the person falling: (i) The person is not aware of the fall and only passive elements of the body help to absorb the impacts. Given that every fall has a great potential for causing important body damage, there is strong evidence that the very nature of human joints, modeled with springs and dampers, and soft tissue passively contribute to ameliorate the effects of falls. In addition, medical literature reports that external padding may also help to diminish the effects of the falls; (ii) The person detects when a fall is initiated and responds accordingly. This explains, for example, why wrist fractures are common: limbs are commonly used to change the impact points in order to redistribute the impact zones along the different surfaces of the body and time; and (iii) As showed in martial arts, if the person can predict a fall, then he can take control of the fall and change it into a fluid movement that helps to quickly recover the desired behavior. This is one step further into distributing the fall into different and wider surfaces of the body and time, aiming towards diminishing or even eliminating fall damage.

When fall are considered from the point of view of robots, things change. For example, medical literature does not talk about joint damage but of bone fractures. In robots it is more plausible that the opposite is more important: it is always possible to build very strong limbs, while the motors in the joints are the ones that suffer damage during impacts. In this sense there has been work done that points out the problems that need to be solved in order to map human movement into robot movement [9].

In general, the study of human falls suggests that for robots it is important to: (i) Design a body that passively helps as much as possible to walk and to fall. A body that uses joint models with springs and dampers as movable parts that act like soft tissue, and padding specially designed to protect important, frail, and/or expensive parts; (ii) Detect a fall as soon as possible to trigger fall-related movements that allows reducing the fall damage; and (iii) If possible, predict falls and redesign normal moves in order to lower the probability of a fall, or to simply control a fall in order to eliminate it as much as possible or to reduce the damage. This work is directly related with the second and third point.

\section{Design of Fall Sequences That Minimize Robot Damage}

Let us consider a humanoid robot with $n$ rotational articulations/joints $q_{i}$. Each articulation is composed by a DC motor, a gearbox and mechanical elements that fix these components (e.g. an articulation built up using a standard servomotor). In this model each articulation can rotate in a given angular operational range:

$$
\theta_{i}^{\min } \leq \theta_{i} \leq \theta_{i}^{\max } ; i=1, \ldots, n
$$




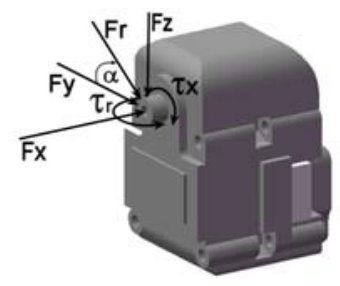

Fig. 1. External forces and torques that can damage an articulation, illustrated for the case of a Dynamixel DX117 motor

The dynamics of each joint $i$ can be characterized in terms of the forces and torques applied in the different axes. Due to the symmetry of the joints, only the axial and radial forces need to be considered. The axial force $F_{a}=F_{x}$ and the magnitude of the radial force $F_{r}=\sqrt{F_{y}^{2}+F_{z}^{2}}$ (see fig. 1) are external forces that can damage the articulation due to impacts produced during a fall. The rotational torque $\tau_{r o t}=\tau_{x}$ is an external torque applied in the direction of rotation of the joint, while the radial torque $\tau_{\text {rad }}=\tau_{r}$ is the torque produced by the radial force. These forces and torques can be produced directly by the fall impacts or be transmitted by the robot body to the articulation. In the short period of time after an impact, a joint can be damaged if the linear or angular impulses (i.e. the integral of each external forces or torques over the time period) surpass a given magnitude that depends on the physical properties of the articulation (motor characteristics, gear material, etc.). Let us define $J_{f a}, J_{f r}, J_{r o t}$ and $J_{r a d}$ as the impulses produced by the axial force, radial force, rotational torque, and radial torque respectively. The damage can be avoided if the following relations hold:

$$
J_{i, f a} \leq J_{i, f a}^{\max } ; J_{i, f r} \leq J_{i, f r}^{\max } ; J_{i, \text { rot }} \leq J_{i, \text { rot }}^{\max } ; J_{i, \text { rad }} \leq J_{i, \text { rad }}^{\max } ; i=1, \ldots, n
$$

with $J_{i, f a}^{\max }, J_{i, f r}^{\max }, J_{i, r o t}^{\max }, J_{i, \text { rad }}^{\max }$ threshold values that depends on the physical properties of the joint.

In addition to the joints' damage, the robot body (mainly frames) can be damaged if the intensity of the fall surpasses a given threshold. Therefore, we need a global measure of the fall intensity. In the biomechanics literature and in studies about falls in humans the impact velocity $v_{i m p}$ is used as a measure of the fall intensity. From the physics point of view, in rigid body collisions the damage is produced by the change of momentum of the colliding objects. Given that in our case collisions are produced between the robot and the ground, which has a much larger mass than the robot, we can assume that the impact velocity is an adequate measure of the fall impact. Hence, to avoid robot body damage, the following should hold:

$$
v_{\text {imp }} \leq v_{\text {imp }}^{\max }
$$

with $v_{i m p}^{\max }$ the maximal impact velocity that do not produce damage in the robot.

Naturally, (2) and (3) are related because the change of the total momentum, which depends on the impact velocity, is equal to the total impulse. This total impulse is then 
propagated through the robot body, producing local impulses in the joints. An important additional requirement to avoid the robot damage is that valuable body parts (CPU, cameras, etc.) should be protected. We assume that these parts will be protected if they do not touch the ground or if they touch it at a low speed. Let us consider $K$ valuable body parts, then the following constraints should hold:

$$
p_{z}^{k}>0 \vee v_{z}^{k} \leq v_{k, z}^{\max } ; k=1, . ., K
$$

with $p_{z}^{k}$ and $v_{z}^{k}$ the vertical position and speed of each valuable part, respectively.

Let us define the joints' positions during the whole fall period as $\Theta_{T_{\text {Fall }}}=\{\Theta(t)\}_{t=0, \ldots, T_{\text {Fall }}}$, with $\Theta(t)$ a vector containing the joints' positions at time step $t$ and $T_{F a l l}$ the fall period. The process of designing a fall sequence is modeled as a search for the $\Theta_{T_{F a l l}}$ that minimizes the damage produced by the fall in robot's joints, frames and valuable parts. From (1)-(4), the expression to be minimized, for a given $\Theta_{T_{\text {Fall }}}$, is given by:

$$
f\left(\Theta_{T_{\text {Fall }}}\right)=\sum_{i=1}^{n} \alpha_{i 1} J_{i, f a}+\alpha_{i 2} J_{i, f r}+\alpha_{i 3} J_{i, \text { rot }}+\alpha_{i 4} J_{i, \text { radial }}+\beta v_{i m p}
$$

subject to

$$
\begin{aligned}
& J_{i, f a} \leq J_{i, f a}^{\max } ; J_{i, f r} \leq J_{i, f r}^{\max } ; J_{i, \text { rot }} \leq J_{i, \text { rot }}^{\max } ; J_{i, \text { rad }} \leq J_{i, \text { rad }}^{\max } ; i=1, \ldots, n \\
& p_{z}^{k}(t)>0 \vee v_{z}^{k}(t) \leq v_{k, z}^{\max } ; k=1, \ldots, K ; t=0, \ldots, T_{\text {Fall }} \\
& \theta_{i}^{\min } \leq \theta_{i}(t) \leq \theta_{i}^{\max } ; i=1, \ldots, n ; t=0, \ldots, T_{\text {Fall }}
\end{aligned}
$$

with $\alpha_{i j}$ weight factors that depends on the importance of each joint (e.g. the neck joint is far more important than a finger joint for a human), on its mechanical properties (e.g. the specific motor model used in the joint), and on the importance of the different forces and torque impulses for the specific joint. $\beta$ is the weighting factor of the global impact measure and $n$ the number of joints. It should be noted that in (5), the force and torque impulses should be measured in the short period of time after an impact.

As already explained, the process of designing a fall sequence consists on searching for a $\Theta_{T_{F a l l}}$ that minimizes an expression that quantifies the damage (eq. (5)). However, to implement directly this search process is highly complex because: (i) when working directly with real robots a large amount of experiments is required, which would eventually damage the robots, (ii) it requires measuring in each joint two linear and two rotational impulse values, as well as the impact velocity, in real-time (at a rate of few milliseconds), and (iii) the high-dimensionality of the parameter space; the search process requires the determination of the position of each joint during the whole fall period.

The first two problems can be overcome if a realistic simulator is employed for the analysis and design of the fall sequences. Using this computational tool, robot damage due to extensive experiments is avoided. In addition, if the simulator is realistic enough, all physical quantities that need to be known for evaluating (5) can be easily determined. The high dimensionality of the parameter space is the hardest problem to 
be tackled. As a suboptimal design strategy, we propose a human-based design procedure consisting on iteratively applying the following consecutive steps: (i) synthesis of fall sequences using a simulation tool, and (ii) quantitative analysis of the obtained sequences using eq. (5). The proposed procedure consists of the following main components: (i) An interactive tool is employed by a human operator for the synthesis of fall sequences (see example in figure 2). For each frame of the sequence, the designer set up all joints' positions; (ii) Each fall sequence is executed in a realistic simulator, and the global damage function given by (5) is used to evaluate the potential damage in the robot body; and (iii) The seeds of the design process, i.e. initial values for the joints' positions during the whole fall period, are examples of human falls, obtained either from standard videos of falls (e.g. martial arts or human sports) or from data acquired using motion capture equipments (e.g. exoskeletons). In the next section we will describe how the proposed strategy has been used in the design of falls sequences for Nao robots.

\section{Experimental Results}

To validate the proposed strategy it was chosen the problem of designing falls for Nao robots [36]. This robot was selected for the following reasons: (i) the robot correspond to an humanoid robot with 22 degrees of freedom (see table 1 for details about the joints and their motion ranges), which represents a complex problem from the point of view of designing fall sequences, (ii) it exists a realistic simulator available for this robot (Webots [34]), (iii) the simulator and robot controller are URBI-compatible [35], which allows building an interactive interface for designing falls, without modifying the simulator or accessing to its source code, and (iv) we have a team in the SPL league (this league use Nao robots), that already classified for the 2008 RoboCup world-competition.

The first step was to build a user interface that allow designing the falls, i.e. specifying the joints positions for the whole frame period $\left(\Theta(t) ; t=0, \ldots, T_{\text {EndFall }}\right)$ and measuring the velocity and impulses values of the falls. This interface was built using URBI. Figure 2 shows its appearance. It can be seen that: (i) the main window has the values of all joint positions for the current frame; these values can be modified by the designer (user), (ii) the bottom window contains the commands for executing the fall, either continuously or in frame-to-frame mode, and (iii) the right window has the values of the radial, axial and angular impulses, the impact velocity, and flags that indicates if any of these values have surpassed their maximal thresholds. In addition, some flags indicate if valuable parts, camera and CPU in this case, touch the floor. The user employs this tool in the designing process, and he can see simultaneously the resulting fall sequence directly in the main simulator window (see examples in fig. 3).

For the purpose of showing the potentiality of this fall designing approach, two "bad" fall sequences and three "good" fall sequences were designed; "good"/"bad" means low/high damage. The designed sequences are: FrontHead: frontal fall where the robot impacts the floor with its face; FrontHand: frontal fall where the only robot action is to put its arm, in a rigid position, to avoid touching the floor with its face; FrontLow: frontal fall where the robot folds its legs in order to lower its center of mass before the impact; FrontTurn: frontal fall where the robot turn its body before 
Table 1. Nao's joint names and motion ranges

\begin{tabular}{|c|c|c|c|}
\hline Body Part & Joint Name & Motion & Range (degrees) \\
\hline \multirow[t]{2}{*}{ Head } & HeadYaw & Head joint twist $(\mathrm{Z})$ & -120 to 120 \\
\hline & HeadPitch & Head joint front \& back (Y) & -45 to 45 \\
\hline \multirow{4}{*}{ Left arm } & LShoulderPitch & Left shoulder joint front \& back (Y) & -120 to 120 \\
\hline & LShoulderRoll & Left shoulder joint right \& left (Z) & 0 to 95 \\
\hline & LElbowRoll & Left shoulder joint twist $(\mathrm{X})$ & -120 to 120 \\
\hline & LElbowYaw & Left elbow joint $(\mathrm{Z})$ & 0 to 90 \\
\hline \multirow{6}{*}{ Left leg } & LHipYawPitch & Left hip joint twist $\left({\left.\mathrm{Z} 45^{\circ}\right)}\right.$ & -90 to 0 \\
\hline & LHipPitch & Left hip joint front \& back (Y) & -100 to 25 \\
\hline & LHipRoll & Left hip joint right and left $(\mathrm{X})$ & -25 to 45 \\
\hline & LKneePitch & Left knee joint $(\mathrm{Y})$ & 0 to 130 \\
\hline & LAnklePitch & Left ankle joint front \& back (Y) & -75 to 45 \\
\hline & LAnkleRoll & Left ankle joint right \& left $(\mathrm{X})$ & -45 to 25 \\
\hline \multirow{6}{*}{ Right leg } & RHipYawPitch & Right hip joint twist $\left(\mathrm{Z} 5^{\circ}\right)$ & -90 to 0 \\
\hline & RHipPitch & Right hip joint front and back (Y) & -100 to 25 \\
\hline & RHipRoll & Right hip joint right \& left $(X)$ & -45 to 25 \\
\hline & RKneePitch & Right knee joint $(\mathrm{Y})$ & 0 to 130 \\
\hline & RAnklePitch & Right ankle joint front \& back (Y) & -75 to 45 \\
\hline & RAnkleRoll & Right ankle right \& left $(\mathrm{X})$ & -25 to 45 \\
\hline \multirow{4}{*}{ Right arm } & RShoulderPitch & Right shoulder joint front \& back (Y) & -120 to 120 \\
\hline & RShoulderRoll & Right shoulder joint right \& left $(\mathrm{Z})$ & -95 to 0 \\
\hline & RElbowRoll & Right shoulder joint twist $(\mathrm{X})$ & -120 to 120 \\
\hline & RElbowYaw & Right elbow joint (Z) & -90 to 0 \\
\hline
\end{tabular}
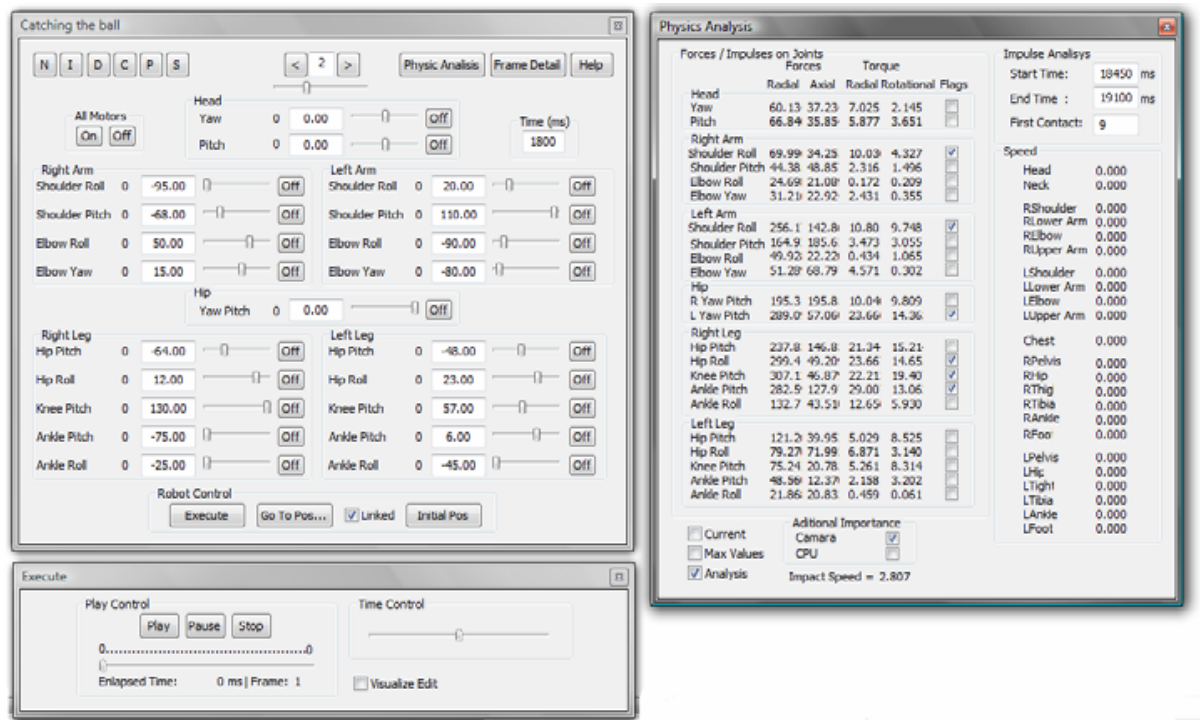

Fig. 2. Image capture of the interactive tool used for the falls design

touching the floor; and BackLow: back fall where the robot separates and folds its legs in order to lower its center of mass before the impact. Videos of all sequences are available in [37]. Two of these sequences are exemplified in figure 3. 


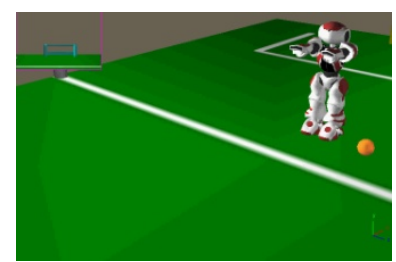

(a1)

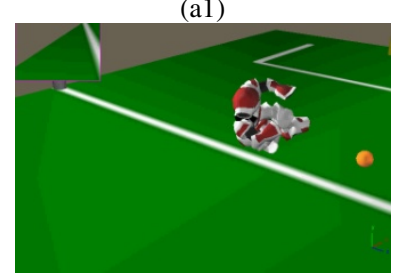

(a4)

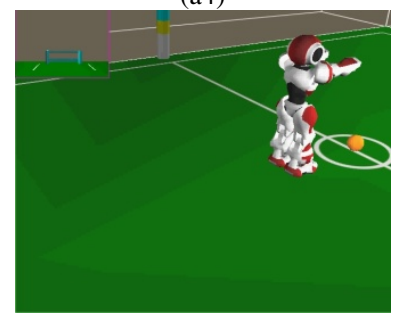

(b1)

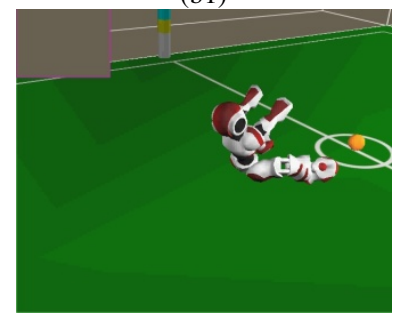

(b4)

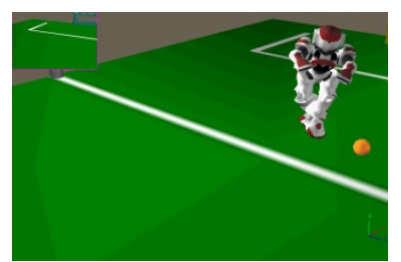

(a2)

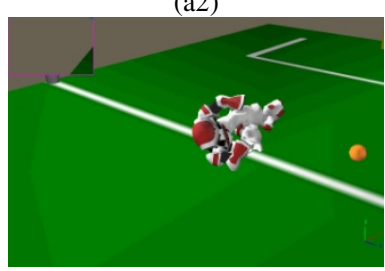

(a5)

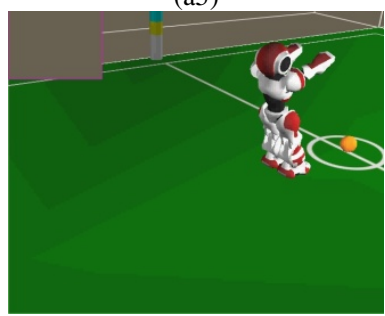

(b2)

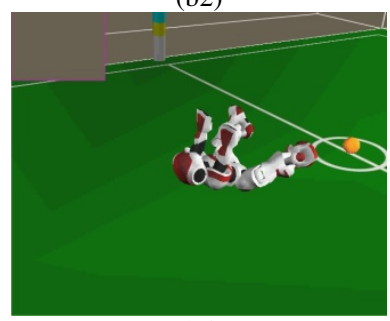

(b5)

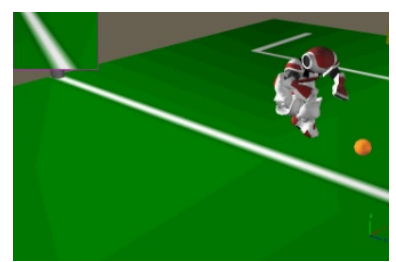

(a3)

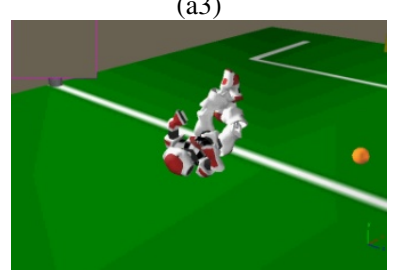

(a6)

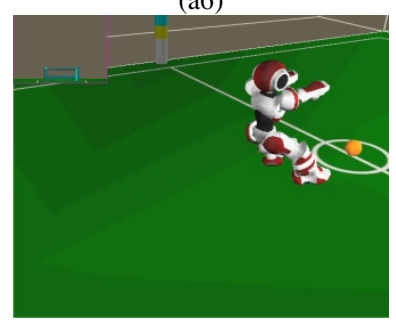

(b3)

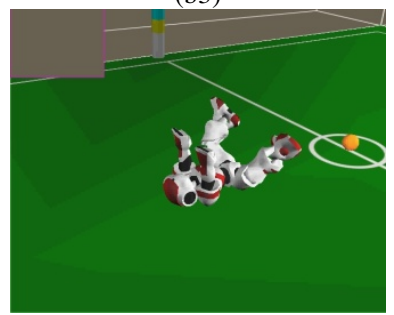

(b6)

Fig. 3. Simulation sequences showing selected frames of the falls under analysis. FrontTurn: (a1)-(a6). BackLow: (b1)-(b6).

It is important to stress that the process of finding "good" falls is a very difficult one, due to the huge size of the search space. In our case the FrontTurn fall was designed by seeing videos of martial arts, while the FrontLow and BackLow falls were designed analyzing data generated by a motion capture exoskeleton (Gypsy-5 from Animazoo [33]).

In tables 2 and 3 quantitative measurements of the fall sequences under analysis are shown. Table 2 contains global measures about the intensity of the impact: velocity, kinetic energy and potential energy before (B) and after (A) the impact. In addition, for each case, the body part that first touches the floor (Body Part), the fall duration in frames ( $\triangle$ Frame), the number of body contact points during the whole fall sequence $\left(T N_{c p}\right)$, and an index consisting in the sum of frames during which the contact points touched the floor $(C P F)$, are shown. Table 3 shows, for each fall 
Table 2. Global measures of the falls under comparison. $\Delta$ Frame: fall duration in frames. $\mathrm{TN}_{\mathrm{cp}}$ : total number of contact point during the fall. CPF: sum of all the contact points times the number of frames that each point is in contact with the ground. Body Part: Part that first contact the body. B: Before collision. A: After collision. K Energy: Kinetic energy. P Energy. Potential Energy.

\begin{tabular}{|l|c|c|c|c|c|c|c|c|c|c|}
\hline \multirow{2}{*}{ Fall name } & \multirow{2}{*}{$\Delta$ Frame } & \multirow{2}{*}{$\mathbf{T N}_{\mathbf{c p}}$} & \multirow{2}{*}{ CPF } & \multirow{2}{*}{ Body Part } & \multicolumn{2}{|c|}{ Velocity $(\mathbf{m} / \mathbf{s})$} & \multicolumn{2}{|c|}{ K Energy $(\mathbf{J})$} & \multicolumn{2}{|c|}{ P Energy $(\mathbf{J})$} \\
\cline { 6 - 11 } & & & & & $\mathrm{B}$ & $\mathrm{A}$ & $\mathrm{B}$ & $\mathrm{A}$ & $\mathrm{B}$ & $\mathrm{A}$ \\
\hline FrontHead & 6 & 1 & 6 & Head & 2.28 & 0.15 & 0.91 & 0.004 & 0.07 & 0.25 \\
\hline FrontHand & 6 & 6 & 26 & LlowerArm & 2.33 & 0.07 & 0.40 & 0.001 & 0.21 & 0.28 \\
\hline FrontLow & 8 & 12 & 37 & RHip & 1.16 & 0.38 & 0.08 & 0.009 & 0.20 & 0.20 \\
\hline BackLow & 8 & 7 & 26 & RHip & 1.11 & 0.63 & 0.07 & 0.02 & 0.26 & 0.20 \\
\hline FrontTurn & 13 & 9 & 19 & RHip & 1.38 & 0.30 & 0.11 & 0.005 & 0.01 & 0.05 \\
\hline
\end{tabular}

Table 3. Maximal values of the axial and radial force impulses, and the torque impulse, for the fall sequences under analysis. In each case, the value of the three largest impulses in $\mathrm{kg} \mathrm{m} / \mathrm{s}$ and the articulation where this impulse was produced, are indicated.

\begin{tabular}{|c|c|c|c|c|c|c|}
\hline & Max. Values & FrontLow & BackLow & FrontTurn & FrontHand & FrontHead \\
\hline \multirow{3}{*}{ 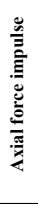 } & First max & $\begin{array}{c}66.9 \\
\text { (RHipYawPitch) }\end{array}$ & $\begin{array}{c}129.4 \\
\text { (RHipYawPitch) }\end{array}$ & $\begin{array}{c}113.6 \\
\text { (RHipYawPitch) }\end{array}$ & $\begin{array}{c}173.9 \\
\text { (RHipYawPitch) }\end{array}$ & $\begin{array}{c}216.8 \\
\text { (RAnkleRoll) }\end{array}$ \\
\hline & Second max & $\begin{array}{c}63.2 \\
\text { (LHipYawPitch) }\end{array}$ & $\begin{array}{c}127.8 \\
\text { (LHipYawPitch) }\end{array}$ & $\begin{array}{c}73.0 \\
\text { (RHipPitch) }\end{array}$ & $\begin{array}{c}173.2 \\
\text { (LHipYawPitch) }\end{array}$ & $\begin{array}{c}214.9 \\
\text { (LAnkleRoll) }\end{array}$ \\
\hline & Third max & $\begin{array}{c}55.3 \\
\text { (LHipRoll) }\end{array}$ & $\begin{array}{c}69.6 \\
\text { (HeadYaw) }\end{array}$ & $\begin{array}{c}69.3 \\
\text { (LHipYawPitch) }\end{array}$ & $\begin{array}{c}104.1 \\
\text { (LShoulderRoll) }\end{array}$ & $\begin{array}{c}211.0 \\
\text { (HeadYaw) }\end{array}$ \\
\hline \multirow{3}{*}{ 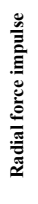 } & First max & $\begin{array}{c}121.8 \\
\text { (LHipPitch) }\end{array}$ & $\begin{array}{c}273.6 \\
\text { (RHipRoll) }\end{array}$ & $\begin{array}{c}222.0 \\
\text { (RHipRoll) }\end{array}$ & $\begin{array}{c}379.2 \\
\text { (LAnkleRoll) }\end{array}$ & $\begin{array}{c}484.3 \\
\text { (HeadPitch) }\end{array}$ \\
\hline & Second max & $\begin{array}{c}118.5 \\
\text { (RKneePitch) }\end{array}$ & $\begin{array}{c}267.1 \\
\text { (LHipRoll) }\end{array}$ & $\begin{array}{c}194.6 \\
\text { (RHipPitch) }\end{array}$ & $\begin{array}{c}378.2 \\
\text { (RAnkleRoll) }\end{array}$ & $\begin{array}{c}414.1 \\
\text { (RAnklePitch) }\end{array}$ \\
\hline & Third max & $\begin{array}{c}115.9 \\
\text { (LKneePitch) }\end{array}$ & $\begin{array}{c}234.2 \\
\text { (RAnkleRoll) }\end{array}$ & $\begin{array}{c}157.2 \\
\text { (LKneePitch) }\end{array}$ & $\begin{array}{c}374.0 \\
\text { (LAnklePitch) }\end{array}$ & $\begin{array}{c}407.0 \\
\text { (LAnklePitch) }\end{array}$ \\
\hline \multirow{3}{*}{ 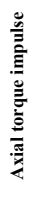 } & First max & $\begin{array}{c}5.7 \\
\text { (RShoulderPitch) }\end{array}$ & $\begin{array}{c}8.5 \\
\text { (LShoulderPitch) }\end{array}$ & $\begin{array}{c}10.7 \\
\text { (LHipYawPitch) }\end{array}$ & $\begin{array}{c}19.1 \\
\text { (LKneePitch) }\end{array}$ & $\begin{array}{c}42.5 \\
\text { (LHipPitch) }\end{array}$ \\
\hline & Second max & $\begin{array}{c}5.4 \\
\text { (RKneePitch) }\end{array}$ & $\begin{array}{c}8.5 \\
\text { (RShoulderPitch) }\end{array}$ & $\begin{array}{c}8.5 \\
\text { (LHipPitch) }\end{array}$ & $\begin{array}{c}19.1 \\
\text { (LHipPitch) }\end{array}$ & $\begin{array}{c}42.5 \\
\text { (LKneePitch) }\end{array}$ \\
\hline & Third max & $\begin{array}{c}5.3 \\
\text { (LKneePitch) }\end{array}$ & $\begin{array}{c}7.7 \\
\text { (RHipRoll) }\end{array}$ & $\begin{array}{c}7.6 \\
\text { (RKneePitch) }\end{array}$ & $\begin{array}{c}18.2 \\
\text { (RKneePitch) }\end{array}$ & $\begin{array}{c}41.9 \\
\text { (RHipPitch) }\end{array}$ \\
\hline
\end{tabular}

sequence, the three maximal impulse's values produced by the radial and axial forces and the external torque, and the articulation where these maxima are produced.

From the results displayed in table 2 we can conclude that, modulating a fall in order to spread the impacts into more contact points and longer periods of time effectively decrease the velocities and energies involved. In addition, a lowering of the center of mass, as in the case of the FrontLow, BackLow and FrontTurn falls, helps to decrease the impact velocity. In table 3 it can be observed that maximal impulses are much lower of the case of longer falls sequences, with several contact points and a lowering in the center of mass. For instance, in the case of the FrontHead fall, the maximal rotational impulse is between 4 and 7.5 times larger than the rotational impulse produced in the good sequences (FrontLow, BackLow, and 
FrontTurn). For the same fall, the maximal linear impulses are at least, two times larger than the ones of the good sequences. A similar situation can be observed for the case of the FrontHand fall. In addition, given the fact that good falls sequences were inspired by the way in which humans falls, good fall sequences tend to concentrate the largest impulses, in articulation that are robust for humans: hip, knee and shoulder.

\section{Conclusions}

In this paper, a methodology for designing fall sequences that minimize the robot damage was presented. This methodology includes the use of a realistic simulator for the design process, and an interactive tool that allows the human designer to select the fall sequence parameters (joints values, sequences extension, etc.), and to observe indices that indicate the quality of the obtained sequence.

Simulations using a 22 DOF humanoid robot show that modeling falls after what is observed in humans greatly decreases the robot damage. Thus, longer falls sequences, with several contact points, and a lowering of the center of mass produce less damage in the robot. In addition, fall sequences that protect valuable body parts, as the head, can also be designed using the proposed methodology. As a future work, we would like to: (i) make a more extensive analysis of the fall sequences, in particular to study the incidence of the impulse forces in the articulations, (ii) to use the designed fall sequences in our humanoid robots, in real soccer games, and (iii) to advance in the automation of the fall design process by using motion capture devices.

\section{References}

1. Baltes, J., McGrath, S., Anderson, J.: The use of gyroscope feedback in the control of the walking gaits for a small humanoid robot. In: Nardi, D., Riedmiller, M., Sammut, C., Santos-Victor, J. (eds.) RoboCup 2004. LNCS, vol. 3276, pp. 628-635. Springer, Heidelberg (2005)

2. Fujiwara, K., Kanehiro, F., Kajita, S., Kaneko, K., Yokoi, K., Hirukawa, H.: UKEMI: falling motion control to minimize damage to biped humanoid robot. In: Proc. 2002 IEEE/RSJ Int. Conference on Intelligent Robots and System, vol. 3, pp. 2521-2526 (October 2002)

3. Fujiwara, K., Kanehiro, F., Saito, H., Kajita, S., Harada, K., Hirukawa, H.: Falling Motion Control of a Humanoid Robot Trained by Virtual Supplementary Tests. In: Proc. 2004 IEEE Int. Conf. on Robotics and Automation, pp. 1077-1082 (2004)

4. Höhn, O., Gacnik, J., Gerth, W.: Detection and Classification of Posture Instabilities of Bipedal Robots. In: Proc. of the 8th Int. Conf. on Climbing and Walking Robots and the Support Technologies for Mobile Machines - CLAWAR, pp. 409-416 (2005)

5. Morisawa, M., Kajita, S., Harada, K., Fujiwara, K., Fumio Kanehiro, K.K., Hirukawa, H.: Emergency Stop Algorithm for Walking Humanoid Robots. In: Proc. 2005 IEEE/RSJ Int. Conf. on Intelligent Robots and Systems, pp. 2109-2115 (2005)

6. Renner, R., Behnke, S.: Instability Detection and Fall Avoidance for a Humanoid using Attitude Sensors and Reflexes. In: Proc. 2006 IEEE/RSJ Int. Conf. on Intelligent Robots and Systems, pp. 2967-2973 (October 2006) 
7. Pagilla, P.R., Yu, B.: An Experimental Study of Planar Impact of a Robot Manipulator. IEEE/ASME Trans. on Mechatronics 9(1), 123-128 (2004)

8. Kellog International Work Group on the Prevention of Falls by the Elderly. The Prevention of Falls in Later Life, Danish Medical Bulletin 34(4), 1-24 (1987)

9. Lopes, M., Santos-Victor, J.: A Developmental Roadmap for Learning by Imitation in Robots. IEEE Trans. on Systems, Man, and Cybernetics-Part B: Cybernetics 37(2), 159 168 (2007)

10. King, M.B.: Evaluating the Older Person Who Falls. In: Masdeu, J.C., Sudarsky, L., Wolfson, L. (eds.) Gait Disorders of Aging: Falls and Therapeutics Strategies. LippincottRaven Publishers (1997)

11. Nevitt, M.C.: Falls in the Elderly: Risk Factors and Prevention. In: Masdeu, J.C., Sudarsky, L., Wolfson, L. (eds.) Gait Disorders of Aging: Falls and Therapeutics Strategies. Lippincott-Raven Publishers (1997)

12. Rubenstein, L.Z., Josephson, K.R.: Interventions to Reduce the Multifactorial Risks for Falling. In: Masdeu, J.C., Sudarsky, L., Wolfson, L. (eds.) Gait Disorders of Aging: Falls and Therapeutics Strategies. Lippincott-Raven Publishers (1997)

13. Nikanjam, M., Kursa, K., Lehman, S., Lattanza, L., Diao, E., Rempel, E.D.: Finger Flexor Motor Control Patterns during Active Flexion: An In Vivo Tendon Force Study. Human Movement Science 26, 1-10 (2007)

14. Lussanet, M.H.E., Smeets, J.B.J., Brenner, E.: Relative Damping Improves Linear MassSpring Models of Goal-Directed Movements. Human Movement Science 21, 85-100 (2002)

15. Perl, J.: A Neural Network Approach to Movement Pattern Analysis. Human Movement Science 23, 605-620 (2004)

16. Sibella, F., Frosio, I., Schena, F., Borghese, N.A.: 3D Analysis of the Body Center of Mass in Rock Climbing. Human Movement Science 26, 851-852 (2007)

17. Smith, J.D., Martin, P.E.: Walking Patterns Change Rapidly Following Asymmetrical Lower Extremity Loading. Human Movement Science 26, 412-425 (2007)

18. Teulier, C., Delignies: The Nature of the Transition between Novice and Skilled Coordination during Learning to Swing. Human Movement Science 26, 376-392 (2007)

19. Huys, R., Daffertshofer, A., Beek, P.J.: Multiple Time Scales and Subsystem Embedding in The Learning of Juggling. Human Movement Science 23, 315-336 (2004)

20. Rousanoglou, E.N., Boudolod, K.D.: Rhythmic Performance during a Whole Body Movement: Dynamic Analysis of Force-Time Curves. Human Movement Science 25, 393408 (2006)

21. Gittoes, M.J., Brewin, M.A., Kerwin, D.G.: Soft Tissue Contributions to Impact Forces Simulated Using a Four-Segment Wobbling Mass Model of Forefoot-Heel Landings. Human Movement Science 25, 775-787 (2006)

22. Katsumata, H.: A Functional Modulation for Timing a Movement: A Coordinative Structure in Baseball Hitting. Human Movement Science 26, 27-47 (2007)

23. Caljouw, S.R., van der Kamp, J.: Bi-Phasic Hitting with Constraints on Impact Velocity and temporal Precision. Human Movement Science 24, 206-217 (2005)

24. Ertan, H., Kentel, B., Tumer, S.T., Korkusuz, F.: Activation Patterns in Forearm Muscles during Archery Shooting. Human Movement Science 22, 37-45 (2003)

25. Loseby, P.N., Piek, J.P., Barret, N.C.: The Influence of Speed and Force on Bimanual Finger Tapping Patterns. Human Movement Science 20, 531-547 (2001)

26. Groen, B.E., Weerdesteyn, V., Duysens, J.: Martial Arts Fall Techniques Decrease the Impact Forces at the Hip during Sideways Falling. Journal of Biomechanics 40, 458-462 (2007) 
27. Pavey, E.: Ready for Their Close-Ups: Breathing Life into Digitally Animated Faces. IEEE Spectrum Magazine, 42-47 (April 2007)

28. Abe, Y., Popovic, J.: Interactive Animation of Dynamic Manipulation. In: Proc. Eurographics/ACM SIGGRAPH Symposium on Computer Animation (2006)

29. Liu, C.K., Hertzmann, A., Popovic, Z.: Learning Physics-Based Motion Style with Nonlinear Inverse Optimization. ACM Transactions on Graphics 24(3), 1071-1081 (2005)

30. Reil, T., Husbands, P.: Evolution of Central Pattern Generators for Bipedal Walking in a Real-Time Physics Environment. IEEE Trans. on Evolutionary Computation 6(2), 159-168 (2002)

31. Hornby, G.S., Pollack, J.B.: Creating High-Level Components with a Generative Representation for Body-Brain Evolution. Artificial Life 8, 223-246 (2002)

32. Zagal, J., Ruiz-del-Solar, J.: UCHILSIM: A Dynamically and Visually Realistic Simulator for the RoboCup Four Legged League. In: Nardi, D., Riedmiller, M., Sammut, C., SantosVictor, J. (eds.) RoboCup 2004. LNCS, vol. 3276, pp. 34-45. Springer, Heidelberg (2005)

33. Animazoo official website, http: / / www . animazoo.com/

34. Webots official website, http: //www. cyberbotics.com/

35. URBI official wbesite, http://www. urbi forge.com/

36. Aldebaran robotics official website, http: / /www. aldebaran-robotics.com/

37. Fall sequences videos, http://www. robocup.cl/fallingvideos 